\title{
230.
}

\section{NOTE SUR LA MÉTHODE D'ÉLIMINATION DE BEZOUT.}

[From the Journal für die reine und angewandte Mathematik, (Crelle), tom. LIII. (1857), pp. 366-367.]

Vorci la forme la plus simple sous laquelle on peut présenter cette méthode. Pour éliminer les variables $x, y$ entre deux équations du $n^{\text {ième }}$ degré

$$
\begin{aligned}
& (a, \ldots \gamma x, y)^{n}=0, \\
& \left(a^{\prime}, \ldots \gamma(x, y)^{n}=0,\right.
\end{aligned}
$$

on n'a qu'à former l'équation identique

$$
\begin{aligned}
& \frac{(a, \ldots \gamma \times, y)^{n}\left(a^{\prime}, \ldots \gamma \lambda, \mu\right)^{n}-\left(a^{\prime}, \ldots \gamma(x, y)^{n}(a, \ldots \gamma \lambda \lambda, \mu)^{n}\right.}{\mu x-\lambda y}=
\end{aligned}
$$

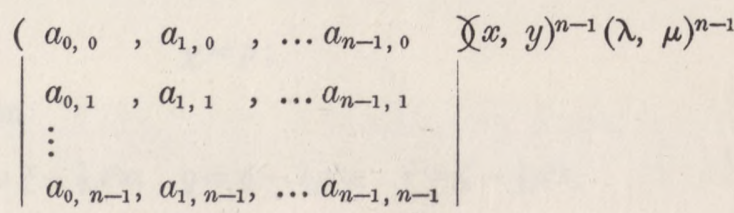

où l'expression qui forme le second membre représente la fonction suivante,

$$
\begin{aligned}
& \left(a_{0,0} \quad x^{n-1}+a_{1,0} \quad x^{n-2} y \ldots+a_{n-1,0} \quad y^{n-1}\right) \lambda^{n-1} \\
& +\left(a_{0,1} \quad x^{n-1}+a_{1,1} \quad x^{n-2} y \ldots+a_{n-1,1} \quad y^{n-1}\right) \lambda^{n-2} \mu \\
& +\left(a_{0, n-1} x^{n-1}+a_{1, n-1} x^{n-2} y \ldots+a_{n-1, n-1} y^{n-1}\right) \mu^{n-1} ;
\end{aligned}
$$


le résultat de l'élimination sera

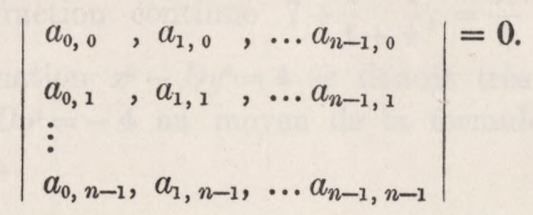

Par exemple on trouve

$$
\begin{aligned}
& \frac{\left(a, b, c \gamma(x, y)^{2}\left(a^{\prime}, b^{\prime}, c^{\prime}\right\rangle \lambda, \mu\right)^{2}-\left(a^{\prime}, b^{\prime}, c^{\prime} \gamma(x, y)^{2}(a, b, c \gamma \lambda, \mu)^{2}\right.}{\mu x-\lambda y}= \\
& \left(\begin{array}{c}
2\left(a b^{\prime}-a^{\prime} b\right), \quad a c^{\prime}-a^{\prime} c \gamma(x, y \gamma \lambda, \mu) ; \\
a c^{\prime}-a^{\prime} c, 2\left(b c^{\prime}-b^{\prime} c\right) \mid
\end{array}\right.
\end{aligned}
$$$$
\frac{(a, b, c, d \gamma x, y)^{3}\left(a^{\prime}, b^{\prime}, c^{\prime}, d^{\prime} \gamma \lambda, \mu\right)^{3}-\left(a^{\prime}, b^{\prime}, c^{\prime}, d^{\prime} \bigvee(x, y)^{3}(a, b, c, d \gamma \lambda, \mu)^{3}\right.}{\mu x-\lambda y}=
$$$$
\left(\begin{array}{ccc}
3\left(a b^{\prime}-a^{\prime} b\right), & 3\left(a c^{\prime}-a^{\prime} c\right), & \left(a d^{\prime}-a^{\prime} d\right) \gamma\left(x^{2}, x y, y^{2} \gamma \lambda^{2}, \lambda \mu, \mu^{2}\right) \\
3\left(a c^{\prime}-a^{\prime} c\right), & \left(a d^{\prime}-a^{\prime} d\right)+9\left(b c^{\prime}-b^{\prime} c\right), & 3\left(b d^{\prime}-b^{\prime} d\right) \\
\left(a d^{\prime}-a^{\prime} d\right), & 3\left(b d^{\prime}-b^{\prime} d\right), & 3\left(c d^{\prime}-c^{\prime} d\right)
\end{array} \mid\right.
$$

d'où l'on tire immédiatement les résultats de l'élimination entre deux équations quadratiques ou cubiques.

Londres, 2 Stone Buildings, Avril, 1855. 
Table des plus petites solutions impaires de l'équation $x^{2}-D y^{2}= \pm 4, D \equiv 5$ (Mod. 8 ).

\begin{tabular}{|c|c|c|c|c|c|c|c|c|c|c|c|}
\hline$D$ & \pm & $x$ & $y$ & $D$ & \pm & $x$ & $y$ & $D$ & \pm & $x$ & $y$ \\
\hline 5 & - & 1 & 1 & 341 & + & 277 & 15 & 677 & & \multicolumn{2}{|c|}{ imposs. } \\
\hline 13 & - & 3 & 1 & 349 & & \multicolumn{2}{|c|}{ imposs. } & 685 & - & $759^{1}$ & 29 \\
\hline 21 & + & 5 & 1 & 357 & + & 19 & 1 & 693 & + & 79 & 3 \\
\hline 29 & - & 5 & 1 & 365 & - & \multicolumn{2}{|c|}{19} & 701 & & \multicolumn{2}{|c|}{ imposs. } \\
\hline 37 & & $\mathrm{imp}$ & Sss. & 373 & & $\operatorname{imp}$ & sss. & 709 & & \multicolumn{2}{|c|}{ imposs. } \\
\hline 45 & + & 7 & 1 & 381 & & \multicolumn{2}{|c|}{ imposs. } & 717 & + & $241^{1}$ & 9 \\
\hline 53 & - & 7 & 1 & 389 & & \multicolumn{2}{|c|}{ imposs. } & 725 & + & 27 & 1 \\
\hline 61 & - & 39 & 5 & 397 & - & 3447 & 173 & 733 & - & 27 & 1 \\
\hline 69 & + & 25 & 3 & 405 & & \multicolumn{2}{|c|}{ imposs. } & 741 & + & 245 & 9 \\
\hline 77 & + & 9 & 1 & 413 & + & 61 & 3 & 749 & + & 12945 & 473 \\
\hline 85 & - & 9 & 1 & 421 & - & 444939 & 21685 & 757 & & \multicolumn{2}{|c|}{ imposs. } \\
\hline 93 & + & 29 & 3 & 429 & + & 145 & 7 & 765 & + & $83^{4}$ & 3 \\
\hline 101 & & \multicolumn{2}{|c|}{ imposs. } & 437 & + & 21 & 1 & 773 & - & 139 & 5 \\
\hline 109 & - & 261 & 25 & 445 & - & 21 & 1 & 781 & & \multicolumn{2}{|c|}{ imposs. } \\
\hline 117 & + & 11 & 1 & 453 & + & 149 & 7 & 789 & + & $31825^{\mathrm{T}}$ & 1133 \\
\hline 125 & - & 11 & 1 & 461 & - & 365 & 17 & 797 & - & 367 & 13 \\
\hline 133 & + & 173 & 15 & 469 & + & 65 & 3 & 805 & + & 1447 & 51 \\
\hline 141 & & imp & ss. & 477 & + & 2599 & 119 & 813 & & \multicolumn{2}{|c|}{ imposs. } \\
\hline 149 & - & 61 & 5 & 485 & & \multicolumn{2}{|c|}{ imposs. } & 821 & - & $16189^{\mathrm{T}}$ & 565 \\
\hline 157 & - & 213 & 17 & 493 & - & $111^{1}$ & 5 & 829 & & \multicolumn{2}{|c|}{ imposs. } \\
\hline 165 & + & 13 & 1 & 501 & + & 28225 & 1261 & 837 & + & $29^{1}$ & 1 \\
\hline 173 & - & 13 & 1 & 509 & - & 925 & 41 & 845 & - & 29 & 1 \\
\hline 181 & - & 1305 & 97 & 517 & + & 10573 & 465 & 853 & - & 27483 & 941 \\
\hline 189 & & \multicolumn{2}{|c|}{ imposs. } & 525 & + & 23 & 1 & 861 & + & 1027 & 35 \\
\hline 197 & & \multicolumn{2}{|c|}{ imposs. } & 533 & - & 23 & 1 & 869 & + & 49377 & 1675 \\
\hline 205 & + & $43^{1}$ & 3 & 541 & - & 1396425 & 60037 & 877 & & \multicolumn{2}{|c|}{ imposs. } \\
\hline 213 & + & 73 & 5 & 549 & + & 1523 & 65 & 885 & & \multicolumn{2}{|c|}{ imposs. } \\
\hline 221 & + & 15 & 1 & 557 & & \multirow{2}{*}{\multicolumn{2}{|c|}{ imposs. }} & 893 & + & 2301 & 77 \\
\hline 229 & - & 15 & 1 & 565 & - & & & 901 & & \multirow{2}{*}{\multicolumn{2}{|c|}{$\begin{array}{l}\text { imposs. } \\
\text { imposs. }\end{array}$}} \\
\hline 237 & + & 77 & 5 & 573 & & \multicolumn{2}{|c|}{ imposs. } & 909 & & & \\
\hline 245 & + & 47 & 3 & 581 & + & $6725^{1}$ & 279 & 917 & + & $1181^{1}$ & 31 \\
\hline 253 & + & 1177 & 74 & 589 & + & 4359377 & 179625 & 925 & & \multicolumn{2}{|c|}{ imposs. } \\
\hline 261 & + & 727 & 45 & 597 & + & 7949 & 399 & 933 & & imp & \\
\hline 269 & & imp & ss. & 605 & + & 123 & 5 & 941 & - & $1135^{\mathrm{Y}}$ & 37 \\
\hline 277 & - & $2613^{1}$ & 157 & 613 & - & 98763 & 3989 & 949 & - & 32685 & 1061 \\
\hline 285 & + & 17 & 1 & 621 & + & 25 & 1 & 957 & + & 31 & 1 \\
\hline 293 & _ & 17 & 1 & 629 & - & 25 & 1 & 965 & - & 31 & 1 \\
\hline 301 & + & 22745 & 1311 & 637 & + & 14159 & 561 & 973 & & imp & \\
\hline 309 & + & 5045 & 287 & 645 & + & 203 & 8 & 981 & + & 68123 & 2175 \\
\hline 317 & - & 89 & 5 & 653 & - & 1661 & 65 & 989 & + & 103245 & 3283 \\
\hline 325 & & imp & & 661 & - & 1789539 & 69605 & 997 & & imp & \\
\hline 333 & & $\operatorname{imp}$ & ss. & 669 & + & 305285 & 11803 & & & & \\
\hline
\end{tabular}

